Research Article

\title{
Manufacturing and Testing of an In Situ Stretching Sample Environment Equipment for Neutron Scattering Experiments
}

\author{
Haibin Chen $\mathbb{D}^{1,2,3}$ Rongyong Li, ${ }^{1,2,3}$ and Yu Liu $\mathbb{D}^{2,3,4}$ \\ ${ }^{1}$ School of Mechanical Engineering, Dongguan University of Technology, Dongguan 523808, China \\ ${ }^{2}$ Neutron Scattering Technical Engineering Research Center, Dongguan University of Technology, Dongnguan 523808, China \\ ${ }^{3}$ Guangdong-Hong Kong-Macao Joint Laboratory for Neutron Scattering Science and Technology, \\ Dongguan University of Technology, Dongguan 523808, China \\ ${ }^{4}$ Spallation Neutron Source Science Center, Dongguan 523808, China
}

Correspondence should be addressed to Yu Liu; liuyu90@ihep.ac.cn

Received 13 August 2020; Revised 10 November 2020; Accepted 18 November 2020; Published 27 November 2020

Academic Editor: Arkady Serikov

Copyright (C) 2020 Haibin Chen et al. This is an open access article distributed under the Creative Commons Attribution License, which permits unrestricted use, distribution, and reproduction in any medium, provided the original work is properly cited.

Neutron scattering technology is one of the most promising ways to observe microstructures of different materials. As a powerful microstructure characterization technology, neutron scattering is widely used in many disciplines. With the help of sample environment equipment, the microstructure detection for materials in various application scenarios can be further realized. In order to detect the microstructure changes of materials under different tensile conditions, an in situ stretching sample environment equipment for neutron scattering experiments was designed and manufactured. Stretching force holding test, sample breaking test, and vacuum maintaining test were carried out. In those tests, a tensile force holding test with no less than 5 hours, a breaking test with a screw bolt as the sample, and a vacuum leakage rate test with no less than 5 hours were obtained, respectively. Through analyzing values obtained, it is shown that the developed prototype of the sample environment equipment is able to meet the experiment requirements. The present prototype provides a reference for further development of sample environment equipment for different application scenarios in neutron scattering experiment.

\section{Introduction}

As a powerful microstructure characterization technology, neutron scattering [1] has been widely applied in many disciplines [2,3]. For some cases to detect microstructures of materials in various application scenarios, a sample environment equipment is required to further improve the capability of the neutron scattering experiment. Among hundreds of neutron scattering experiments carried out each year in different neutron research centers across the world, most of them have employed additional equipment to apply special physical or chemical "condition" or "environment" to introduce the sample into a phase or state of particular interest. Most commonly, the "condition" should be cryogenic, high temperature, high pressure, magnetic field, viscous flow, gas flow, chemical preparation, or sometimes a simultaneous combination of two or more of them together [4].
At present, there are four spallation neutron sources and several reactor- and accelerator-driven neutron sources in the world. Various sample environment equipment are therefore required for the neutron scattering experiments. There are 27 neutron scattering spectrometers in ISIS (spallation neutron source in UK). Typical diffraction spectrometer ENGIN-X has been widely used in materials and machining, where high temperature in situ mechanics experiments in neutron diffraction were realized. By setting a sample environment with heating by infrared ray, the designed maximum temperature, $1100^{\circ} \mathrm{C}$, was reached in the experiment [5]. There are 20 neutron scattering experiments in the first phase of China Spallation Neutron Source (CSNS). According to the application fields, CSNS is divided into the following four departments: quantum and disordered materials, materials science and engineering, soft matter and bioscience, and energy and 
environmental science [6]. Among them, magnetic particle imaging (MPI) spectrometer under construction will be mainly used for the structure analysis of different ordered materials, and the application fields will be mainly focused on the structure research of long-range ordered but shortrange disordered materials or long-range disordered but medium-short-range ordered materials. To this end, in situ stretching sample environment can be used to detect the microstructure of different ordered materials under different stress states.

Stretching or in situ stretching is a common technique in material research. Some high-temperature stretching tests on glass fiber-reinforced composites were carried out, and changing laws of stretching mechanical performances were obtained by Gao et al. [7]. A high-temperature stretching mechanical behavior and failure mechanism research of $(\mathrm{TiBw}+\mathrm{TiCp}) / \mathrm{Ti}$ composite was done by Zhang et al. [8]. Some uniaxial tension tests in AISI 8620, 4320, and 3310 steels about in situ neutron diffraction and crystal plasticity modeling were carried out for explaining the effect of nickel on the kinematic stability of retained austenite in carburized bearing steels by Bedekar et al. [9]. At present, in situ experiments and equipment in neutron scattering are relatively rare, and the reference of in situ stretching samples environment equipment is also less. Sample environment equipment and facilities are not simply accessories to the experiment but are an essential and integral part of it [4]. It needs to work under relatively extreme working conditions, to meet the complex requirements of sample position adjustment and physical operation and to consider the influence of neutron attenuation and scattering background. This fully shows us the manufacturing difficulty and necessity to carry out test and research of the in situ stretching sample environment equipment for neutron scattering experiments.

\section{Design Objectives}

The sample can be stretching-loaded while it is irradiated by the neutron scatting at the same time, with the help of the in situ stretching sample environment equipment in the neutron scattering experiment. Values from the neutron scattering in dynamic stretching are obtained and used to study the microstructure and physical properties of the sample under stress. For the sake of carrying out the above experiments, a set of sample environment equipment meeting the following objectives is designed, with the composition as shown in Figure 1.

First, the addressed equipment should have special stretching mechanism. Remote servo control, stretching value output, and power-off brake self-locking, can provide stable pull load within $30 \mathrm{kN}$. The sample fixture is designed to be fixed by threads. Both ends of the sample are threaded, for which one end of the sample can be screwed into the bottom of the sample rod and the other end can be fixed with a special nut.

Moreover, the designed equipment should have sample rods. Due to the large number of samples to be tested during the experiment, a sample exchanging time is set at $5 \mathrm{~min}$. A number of sample rods should be prepared to achieve the goal that one can be taken out and the other can be inserted quickly after being cooled. Due to the large length and tension force of the sample rod, the material with high strength and good rigidity should be used. This can effectively reduce the diameter and keep the sample aligned with the neutron channel.

Finally, vacuum mechanism should be provided. In order to reduce the loss of neutrons in the air, the flight path of neutrons should be as close to the vacuum as possible. The vacuum inside the equipment should be increased. The extreme vacuum degree of the neutron tube is designed at $10 \mathrm{~Pa}(0.01 \mathrm{MPa})$ [10]. Therefore, the vacuum degree of outer cylinder and inner cylinder in the designed sample environment should be kept below this value. Also, the inner cylinder and outer cylinder should be isolated with vacuum.

\section{Equipment Prototyping}

In situ stretching sample environment prototyping is a comprehensive engineering which integrates mechanical, electrical, and control components together. The manufacturing focus is on stretching mechanism and vacuum mechanism. Among them, the stretching mechanism is the core part of the in situ stretching sample environment, which is mainly composed of fixture, sample rod, tension sensor, gear reducer, and servomotor. The stretching mechanism mainly ensures the stability of tensile force and alignment of neutron channel.

3.1. Standard Components of the Equipment. The servomotor with $750 \mathrm{~W}$ power and $2.37 \mathrm{Nm}$ torque provides controllable stretching force and self-locking function by power-off brake. The gear reducer with a reduction speed ratio of 25 realizes speed reduction, torque increasing, and self-locking function by a stop brake. The force sensor provides the input power measurement, with the maximum measurement value of $50 \mathrm{kN}$. The sample rod is built-in with a screw-rod with diameter $32 \mathrm{~mm}$ and lead $5 \mathrm{~mm}$. To sum up, $63 \mathrm{kN}$ tension force was provided by the stretching mechanism. Compared to $30 \mathrm{kN}$ tension force of the design objectives, 2 times spare coefficient was set up.

3.2. Nonstandard Components Manufactured. As a nonstandard component, the sample rod was made as an independent replaceable part. There are two interfaces of the sample rod: the interface at the vacuum chamber and the interface at the stretching mechanism. The sample fixture is a nut simplifying the sample holding mode. The stretching mechanism is shown in Figure 2.

Another important nonstandard component is the vacuum mechanism composed of inner and outer vacuum cylinders, connecting valves, corrugated pipes, and vacuum pumps. Metal seals are used for the interface, as shown in Figure 3. In order to reduce the absorption of neutrons by air during flight, the tube cavity is usually kept in a good vacuum state [10]. So, the sample environment should be kept close to the vacuum state after receiving the neutron from the tube. 


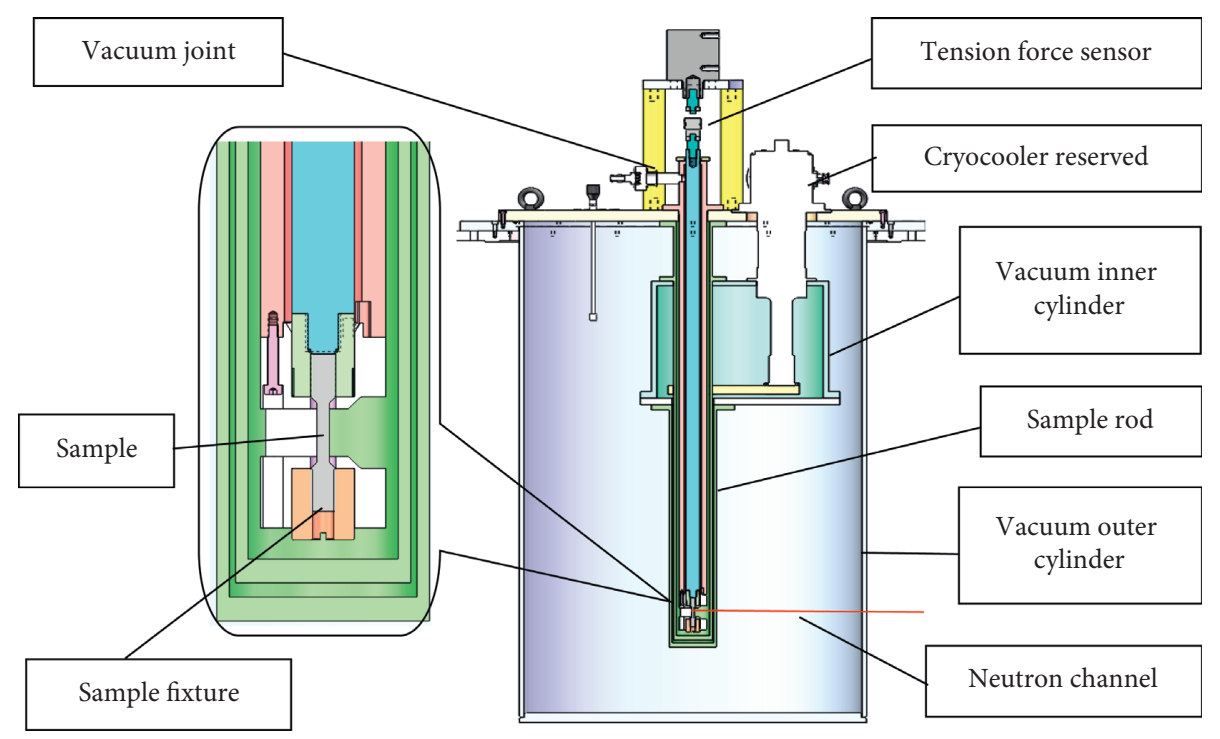

Figure 1: The structure of the in situ stretching sample environment.

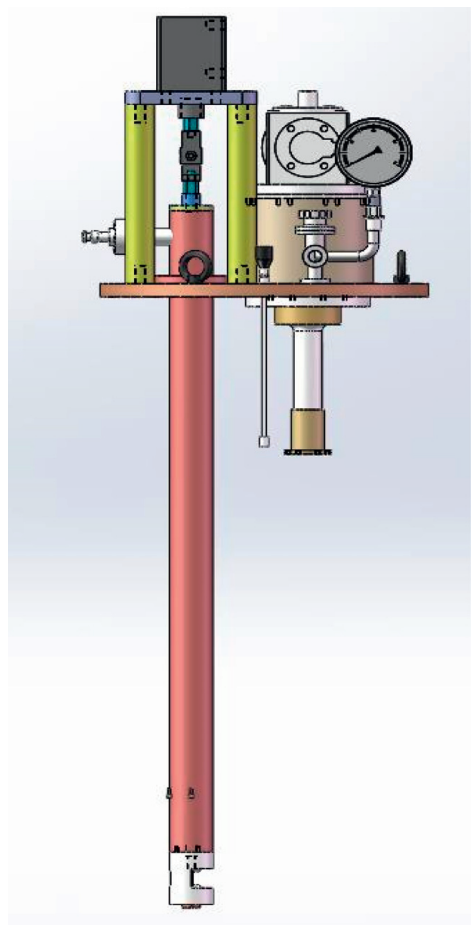

(a)

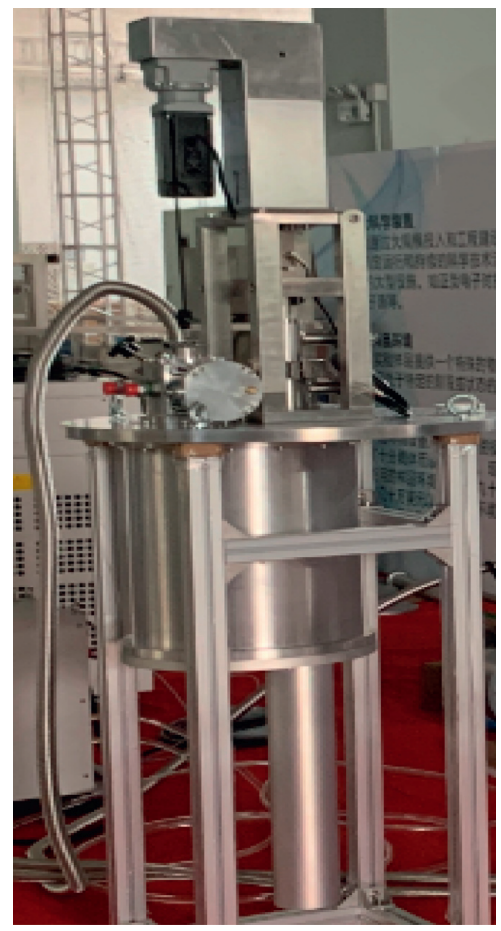

(b)

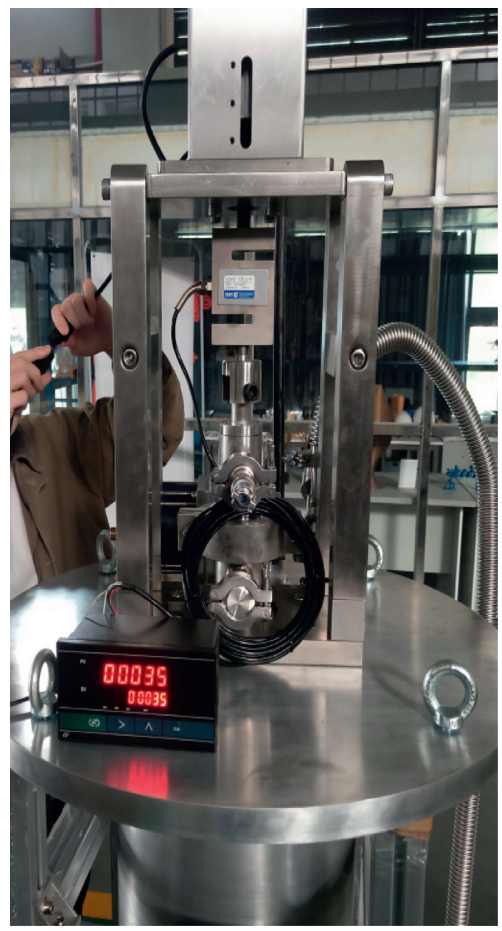

(c)

Figure 2: Prototype of the stretching mechanism.

\section{Tests and Analyses}

To evaluate the performance of the developed prototype device, a sample was prepared for the test. The sample was made with length $30 \mathrm{~mm}$ and M10 thick tooth screw. The material of the sample is stainless steel with strength of 8.8 grade. The theoretical maximum tensile force is $36 \mathrm{KN}$. The vacuum pump was started first. The stretching force holding test, sample breaking test, and vacuum pressure maintaining test were carried out, respectively.
4.1. Stretching Force Holding Test and Analysis. Generally speaking, the scanning time of the neutron scattering experiment is long. Several fixed stretching forces should be provided by the stretching mechanism for a long time for obtaining the microscopic state of the sample under different tensile forces. Therefore, the stretching force holding test was carried out with the stretching force at $1.495 \mathrm{KN}$. The test was maintained for no less than $5 \mathrm{~h}$ by motor power-off brake. The test result is shown in Figure 4. 


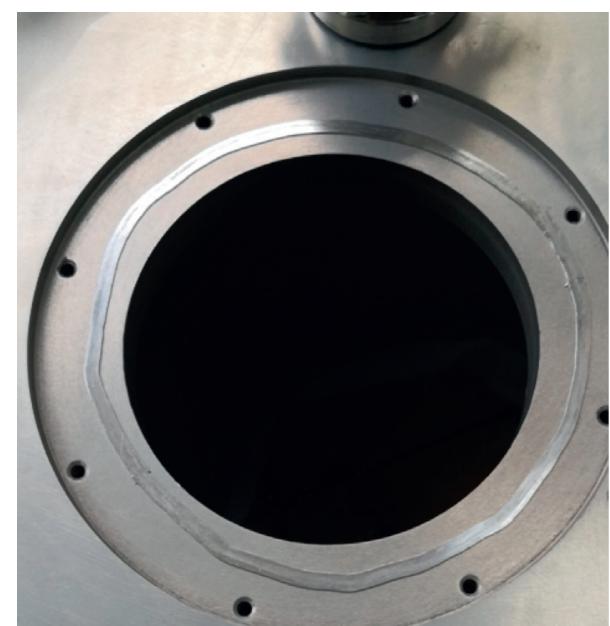

Figure 3: Metal seal in the interface.

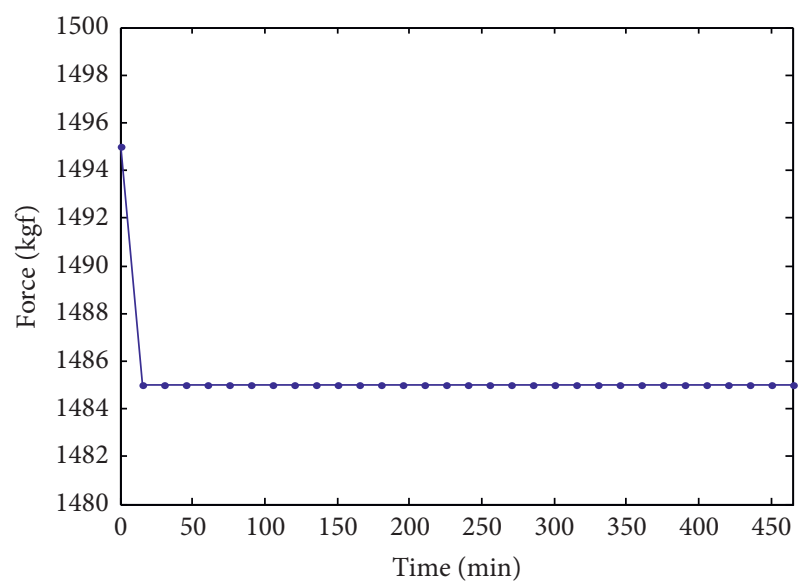

FIgURE 4: Result of the tensile pressure holding test.

As shown in the figure, while the stretching force was loaded to $1.495 \mathrm{kN}$ and stopped for $0.5 \mathrm{~h}$, the stretching force was reduced to $1.485 \mathrm{kN}$. In the forthcoming $5 \mathrm{~h}$, the stretching force loss is almost zero (the values focus on a straight line of $y=1.485 \mathrm{kN}$ ). It fully shows that the selflocking effect is good, and the stretching mechanism can provide a stable tension. This indicates that the stretching force holding has met the design objective.

4.2. Breaking Test and Analysis. According to the aforementioned design objectives, stretching mechanism should be required to reach at least $30 \mathrm{kN}$ stretching force and to stably keep at about $30 \mathrm{kN}$ after stopping the load. Finally, stretching force was continued to increase until the sample was broken. The experiment result is shown in Figure 5.

The stretch force was increased to $30 \mathrm{kN}$ after the $4^{\text {th }}$ loading. The sample was broken in the $16^{\text {th }}$ loading (the stretching force was $34 \mathrm{kN}$ ). This was close to the theoretical maximum stretching force of the sample (i.e., $36 \mathrm{kN}$ ). This was in line with the expected design objective. The broken sample is shown in Figure 6.

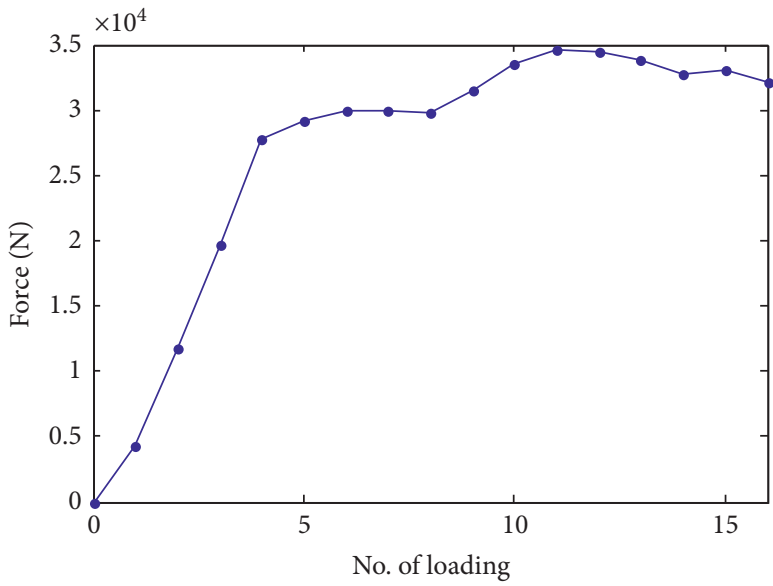

Figure 5: Values in the breaking test.

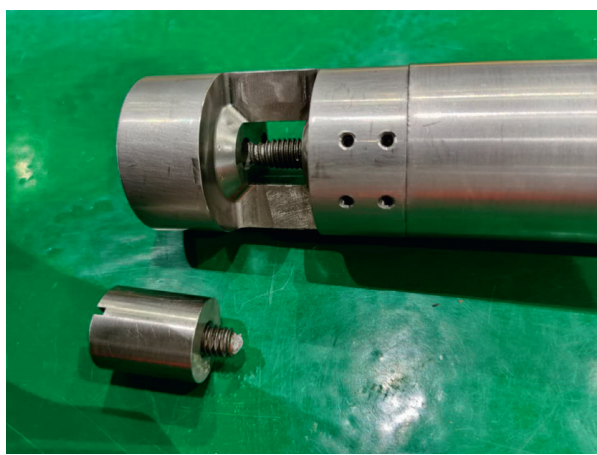

Figure 6: The broken sample.

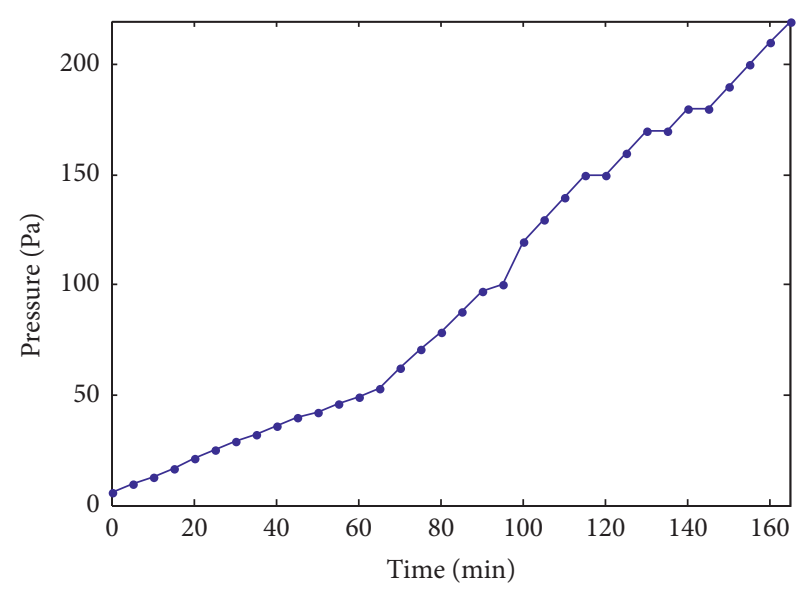

FiguRE 7: Values in vacuum pressure maintaining test.

4.3. Vacuum Pressure Maintaining Test and Analysis. Before the vacuum pressure maintaining test, the vacuum condition inside the sample environment was pumped to $1 \mathrm{~Pa}$ (lower than the design objective $10 \mathrm{~Pa}$ ) as the initial state. After stopping the vacuum pump and keeping the sample environment be airtight, vacuum values was recorded in the coming $5 \mathrm{~h}$, and the experiment values are shown in Figure 7. 
From the analysis of values, the curve formed is close to a straight line, indicating that the leakage rate is close to a constant value of $35 \mathrm{~Pa} / \mathrm{h}$. This is far lower than the pumping rate of the vacuum pump. Therefore, the sealing performance of the vacuum mechanism has met the design objective (less than $10 \mathrm{~Pa}$ ).

\section{Conclusions}

Conclusions can be drawn from the test results and analyses as follows:

(1) During the stretching force test, the stretching force was loaded to $1.495 \mathrm{kN}$ and gradually stabilized to $1.485 \mathrm{kN}$. This almost does not weaken in the following $5 \mathrm{~h}$. It proves that the self-locking effect is good, and the sample environment can meet the design objective.

(2) During the breaking test, the sample was broken at a stretching force of $34 \mathrm{kN}$. It is close to the theoretical maximum tensile force of $36 \mathrm{kN}$, achieving the expected design objective.

(3) During the vacuum pressure maintaining test, the curve formed is close to a straight line, with the leakage rate at the constant value of $35 \mathrm{~Pa} / \mathrm{h}$. It is lower than the pumping rate of the vacuum pump, indicating that the sealing performance of vacuum mechanism meets the design objective.

\section{Data Availability}

All data included in this study are available from the corresponding author upon request.

\section{Conflicts of Interest}

The authors declare that they have no conflicts of interest.

\section{Acknowledgments}

This work was supported in part by the National Natural Science Foundation of China (no. 51775112), the Guangdong Basic and Applied Basic Research Foundation (no. 2019B1515120095), the Scientific Research Foundation of Advanced Talents (Innovation Team) of Dongguan University of Technology (no. KCYCXPT2016004), and the Guandong Province Colleges and Universities Epidemic Prevention and Control Project (no. 2020KZDZX1199).

\section{References}

[1] L. P. Guo and K. Sun, "Monte Carlo simulation on neutron scattering facilities," World Science and Technology $R$ \& $D$, vol. 29, no. 2, pp. 1-7, 2007.

[2] P. G. Xu and T. Yo, "Recent neutron scattering study on heat treatment of materials," Heat Treatment, vol. 21, no. 4, pp. 1-7, 2006.

[3] H. Cheng, W. Zhang, and F. W. Wang, "Applications of the China spallation neutron source," Physics, vol. 48, no. 11, pp. 701-707, 2019.
[4] I. F. Bailey, "A review of sample environments in neutron scattering," Oldenbourg Wissenschaftsverlag, vol. 218, pp. 84-95, 2003.

[5] X. Zhan, J. Kelleher, J. Gao et al., "High temperature sample environment upgrade of ISIS engineering materials in-situ diffraction experiment," Acta Physica Sinica-Chinese Edition, vol. 68, no. 13, Article ID 132901, 2019.

[6] H. Chen, "China spallation neutron source," Modern Physics, vol. 1, no. 1, pp. 3-10, 2016.

[7] Y.-H. Gao, Y. Tian, Q.-P. Jin, and Z.-G. Lin, "Effect of temperature on tensile properties of GFRP bars," China Plastics Industry, vol. 44, no. 9, pp. 95-99, 2016.

[8] C. Zhang, S. Zhang, Z. Hou et al., "Tensile mechanical behavior and failure mechanism of ( $\mathrm{TiBw}+\mathrm{TiCp}) / \mathrm{Ti}$ composites at elevated temperature," The Chinese Journal of Nonferrous Metals, vol. 26, no. 11, pp. 2287-2295, 2016.

[9] V. Bedekar, R. Voothaluru, D. Yu et al., "Effect of nickel on the kinematic stability of retained austenite in carburized bearing steels in-situ neutron diffraction and crystal plasticity modeling of uniaxial tension tests in AISI 8620, 4320 and 3310 steels," International Journal of Plasticity, vol. 131, Article ID 102748, 2020.

[10] Y. Li, K. Sun, Y. Wang et al., "Conceptual design of thermal neutron guide of neutron diffractometer for residual stress field and defect in material and component," Atomic Energy Science and Technology, vol. 54, no. 1, pp. 158-165, 2020. 